\title{
Principles and Experimental Methods for Underwater Concrete Formulations
}

\author{
Kiran Dabral ${ }^{1}$, R Selvaraj ${ }^{2}$, Simon $\mathrm{J}^{3}$ \\ ${ }^{1}$ M.Tech Structural Engineering Student, VIT University, Vellore, Tamil Nadu, India, \\ ${ }^{2}$ Principal Scientist, CSIR-Central Electrochemical Research Institute, Karaikudi, Tamil Nadu, India, \\ ${ }^{3}$ Assistant Professor, VIT University, Vellore, Tamil Nadu, India,
}

\begin{abstract}
Underwater concreting is one of the oldest methods of placing concrete under the water for the construction of submerged structures like harbors, bridges, aqueducts and other similar structures. From the literatures, it is found that very few works has been done for promoting the underwater concrete (UWC) at present scenario. There is a global need for preparing the UWC which should have properties to suit the current requirements to withstand environmental needs, strength, anti-washout properties, quick setting and also to have bonding properties with existing old concretes for the repairing purposes. Keeping all these things in mind, 15 formulations comprising of few bio-materials with mineral admixtures are proposed in this paper. Necessary critical tests, especially needed for evaluating the UWC for required durability purposes, are discussed in detail.
\end{abstract}

Keywords: underwater concrete, bio-materials, mineral admixtures, spray test, drop test, anti-washout, flowability, bleeding, setting time

\section{Introduction}

In ancient engineering, Roman builders discovered how to create hydraulic mortar, a building material which could potentially be used for the development of infrastructure of their country. For the Romans, the pozzolonic additive was sand like volcanic ash, pozzolonic materials which are composed of chemically reactive aluminosilicateswhich, when mixed with lime and water, produces a series of hydrated calcium-silicates and aluminates. These compounds caused the hydraulic mortar to set slowly, particularly under water and become extremely hard. Pollio Vitruvius, a Roman published many books on architecture circa $25 \mathrm{BC}$, describing the engineering and building methods practiced during the Roman period. He specified that pozzolona (quarry sand) from the area around Baiac, or from entire coastline of the Bay of Naples, had to be used to produce hydraulic concrete. The Roman structures made use of Pozzolona imported from the Bay of Naples, from the region around Puteoli. In the study of cores of concrete from underwater structures, it appeared that the pozzolona was shifted from the Bay of Naples to provide significant uniformity in maximum grain size. Although the exact ratio of pozzolona to lime in the mortar of the cores remained to be determined, it was clear that the mortar was very carefully measured and mixed. And the aggregates were also carefully selected and sorted by size, and ratio of mortar to aggregate remained very uniform throughout each structure. The remarkable homogeneity of the concrete and the relative absence of voids indicated that the material was well compacted during placement [1].

The present work of construction ofunderwater structures has taken a leap. The procedure of formulating the concrete mixtures should be in such a way that they are easy to place and also withstand even in the adverse underwater conditions. The required properties are- (a)ability of concrete to flow, (b) retention of workability over a reasonabletime, (c) self-compacting, (d)adequate cohesion to avoid segregation, (e) low heat of hydration, (f) low bleeding, (g) controlled set times, (h)development of adequate compressive strength, (i) adequate bond strength, (j) low creep and shrinkage, (k)resistance to washout by flowing water ,(l) abrasion resistance, and some more according to the condition in which the structure is to be built [2].

The methods which are used to place concrete underwater are:(a) the pre-packed concrete method, (b)the tremie method, and(c)the concrete pump method [3]. Use of the tremie is currently the most often utilized technique for placing concrete under water. To meet all the requirements of properties of underwater concrete, researchers have been extensively working on construction material to develop admixtures for use in concrete that permit the concrete to be placed underwater without the use of tremie. These materials are referred to as anti-washout concrete. The admixture is used to prevent washout of cementitious material and dispersion of aggregate during underwater placement of concrete. Hydroxyethylcellulose (HEC), hydroxyethylmethylcellulose (HEMC), and hydroxypropylmethylcellulose(HPMC) are among the various admixtures used.The viscosities of the admixtures differ considerably according to polymerization, molecular weight and type of substituent, when they are dissolved. They dissolve in water rapidly when placed in an alkaline environment such as concrete. 
In our present research work, it is proposedto make some trial mixes using bio-materials and viscosity enhancing natural materials which are inexpensive and are readily available in market. Out of all the mixes, the material which gives the best performance as an anti-washout admixture and optimization of the dosage of that materialfor specific volume of concrete will be carried out.

\section{Experimental Formulations}

It is proposed tocast UWC specimens based on the following formulations and materials:

1. OPC Concrete (Control mix)

2. $\mathrm{OPC}+\mathrm{SP}+\mathrm{MS}+\mathrm{SF}$

3. $\mathrm{OPC}+\mathrm{SP}+\mathrm{VAJRAM}+\mathrm{MS}+\mathrm{SF}$

4. $\mathrm{OPC}+\mathrm{SP}+\mathrm{WATER}$ SOLUBLE ACRYLIC + MS + SF

5. $\mathrm{OPC}+\mathrm{SP}+\mathrm{PISTA} \mathrm{GUM}+\mathrm{MS}+\mathrm{SF}$

6. $\mathrm{OPC}+\mathrm{SP}+\mathrm{JAGGERY}+\mathrm{MS}+\mathrm{SF}$

7. $\mathrm{OPC}+\mathrm{SP}+\mathrm{FEVICOL} \mathrm{RESIN}+\mathrm{MS}+\mathrm{SF}$

8. $\mathrm{OPC}+\mathrm{SP}+\mathrm{ALUMINA} \mathrm{CLAY}+\mathrm{MS}+\mathrm{SF}$

9. $\mathrm{OPC}+\mathrm{SP}+\mathrm{ORDINARY} \mathrm{CLAY}+\mathrm{MS}+\mathrm{SF}$

10. $\mathrm{OPC}+\mathrm{SP}+\mathrm{ACETATES}+\mathrm{MS}+\mathrm{SF}$

11. $\mathrm{OPC}+\mathrm{SP}+\mathrm{MURUNGAI} \mathrm{GUM}+\mathrm{MS}+\mathrm{SF}$

12. $\mathrm{OPC}+\mathrm{SP}+\mathrm{ASAEFOETIDA}+\mathrm{MS}+\mathrm{SF}$

13. $\mathrm{OPC}+\mathrm{SP}+\mathrm{KADUKKAI} \mathrm{LIQUID}+\mathrm{MS}+\mathrm{SF}$

14. OPC + SP + STARCHES (MAIDA, VENDAYAPODI, TAPIACO POWDER) + MS + SF

15. $\mathrm{OPC}+\mathrm{SP}+\mathrm{SUPER}$ ABSORBENT POLYMERS + MS + SF

Where, OPC: Ordinary Portland cement

SP: Super plasticizer

MS: Micro Silica

SF: Silica Fumes

\section{Constituent materials and their influence in UWC}

Influence of cement: Portland cement influences the behavior of fresh concrete in three fundamental ways (a)Cement hydration, refers to chemical and physical processes taking place. Cement containing high $\mathrm{C}_{3} \mathrm{~A}$ content usually causes rapid hydration of cement and consumption of free water in the paste. (b)Water demand, the gradation of cement particles, the $\mathrm{C}_{3} \mathrm{~A}$ content and the alkali content determines the water demand. The finer the cement, the higher the water demands. (c)Cement paste, the high cementitious material content is essential to enhance the cohesion and flowability of concrete, thereby reducing laitance and segregation.

In massive underwater construction, the workability of concrete and the heat of hydration are the two most important concerns. Therefore, type 2 Portland cement is most preferable for use in underwater construction. Blended cement is not recommended.

Influence of cement paste: It is a mixture of cement,mineral binders, fines and water in concrete. Cement paste affects the workability of concrete in three ways: (a) the volume of cement paste, (b) the rheology of cement paste (it describes yield stress and plastic viscosity of concrete), and (c) the interaction between cement paste and aggregates.

An increase in the cement paste often leads to more flowable concrete. If the ratio of water-tocementitious materials is kept constant, increasing cementitious materials generally improves workability of concrete.

Influence of mineral admixtures: Mineral admixtures refer to pozzolonic materials such as fly ash and silica fume. Pozzolona react with the by-products of the cementitious reaction at later stage. For massive underwater construction, adding mineral admixtures to the concrete mixture as partial replacement of Portland cement is important. The performance requirements of high-performance underwater concrete cannot be met without using admixtures.

Proper use of mineral admixtures improves the quality of concrete in all the important aspects. It (a) improves workability, flowability, and pumpability, (b) improves homogeneity and uniformity of concrete mixes, (c) enhancing the resistance to segregation and erosion, (d) lower heat of hydration, (e) low bleeding, and (f) better control of setting time.

Influence of aggregates: Cement paste is the continuous paste that carries aggregates as suspended particles. The higher the friction force, the lower the slump. A concrete containing large and angular aggregate tends to be less workable and often has difficulty flowing through reinforcement cages. High content of fine aggregates 
tend to reduce segregation and bleeding. Modern underwater concrete usually contains fine aggregates in the range of 45 to 50 percent of total aggregates. The percentage of fine aggregates passing $75 \mathrm{micron}$ sieve is recommended to be about 10 percent of the aggregate volume.

The amount of coarse aggregate is measured as the volume ratio of coarse aggregates to the total solids in concrete. A high ratio results in high yield stress and high viscosity. Since underwater concrete should be flowable and self-compacting, this ratio is usually limited within the range of 0.37 to 0.50 .

Influence of water content: Water is the medium that carries aggregates and binders as suspended particles. The water coats and lubricates the suspended particles, resulting in the plasticity and flowability of concrete. The water content in a mix can be classified in two categories: (a) water absorbed in the aggregate, (b) free water that provides workability, and is the amount used in calculating water-cement ratio. The absorbed water generally does not contribute to the workability of concrete. The larger the total surface area of solid particles, the higher the water demand. The physical binding of water is almost directly proportional to the surface area of fines. It is the quantity and quality of free water in a mix that determines rheological properties of fresh concrete. The workability of concrete largely depends on the ratio of water to fines content. In underwater concrete, the ratio of water to fines usually ranges from 0.9 to 1.0 by volume. For given water content, high fines content leads to more cohesive concrete, and thus less bleeding and segregation [2].

\section{Test methods for UWC}

Some of the testing methods used to evaluate the quality of underwater concrete are:

\section{CRD C61 test}

This test uses a small basket with small diameter holes $(3 \mathrm{~mm})$ and the basket full of concrete is immersed in water three times. It is possible to find washout resistance due to the aggregates in the mix by blocking the holes of the basket, using this method. The test was later modified to use a larger basket with a larger diameter of holes $(20 \mathrm{~mm})$. The number of immersions was increased from three to five. This test is also called Plunge test. This test measures the mass loss of concrete by washout in water [4].

\section{Drop test}

The basic equipment constitutes graduated cylinder filled with water. A small quantity of concrete $(400-500 \mathrm{~g})$ is dropped through the water in the cylinder. The resultant turbidity of the water is visually assessed to determine the degree of washout. The degree of turbidity can be measured using turbidity meter [6].

\section{pH factor test}

This procedure involves a beaker filled with water. A fresh concrete sample of an appropriate size is divided into several parts and then dropped into the beaker. It is allowed to settle and is observed after three minutes, and a unit volume of the supernatant solution (the water solution above, when concrete has precipitated at the bottom) is decanted into another beaker. The $\mathrm{pH}$ factor of the solution is determined and recorded. The higher the $\mathrm{pH}$ of the decanted solution, the higher is the washout loss [6].

\section{Spray test}

This test apparatus determines quantitative results of the resistant to washout of a concrete. A mould filled with a sample of $1 \mathrm{~kg}$ of fresh concrete is placed on a base plate. The mould is removed and the base plate with the sample is placed on a frame suspended from an electronic balance. A computer is connected to the balance, and is set in such a way that it automatically switches on recording of the washout process, when there is increase of the pressure on the baseplate by the water spray. The recording begins automatically when the tap on the pipe connecting the water tank with the spray head is opened and waterfrom the spray head washes out the sample for four minutes. A constant head is maintained in the water tank in order to keep the same pressure throughout the test. The measurement recorded directly from the balance every two seconds is the mass of the sample resting on the base plate and mass due to pressure and volume of the water poured onto the sample. The net amount of the material lost is obtained as the effect of the pressure and mass of the poured water is subtracted from the direct measurement from the balance.An expression of the washout is provided as a percentage of the mass lost compared to that of the original mass [5].

\section{Characteristics of Anti-washout UWC}

Anti-washout UWC has slightly different properties than ordinary hydraulic cement concrete because of the effect of the admixture. Fresh UWC can be characterized by following properties: 


\section{Flowability}

Due to the increased viscosity of anti-washout UWC, the slump transformation takes place over several minutes. The slump is ultimately 8 to $10 \mathrm{in}$. To have better understanding of the flowability of this type of concrete, a slump-flow value or a spread value is more suitable than a slump value. In general, slump test is done for stiff UWC. For such types of concrete, very minimal slump to zero slump is also adopted. In case of flowable UWC (self-compacting and flowable mix), a flow table test is conducted as per German standard DIN 1048 , wherein diameter of the flow on the flow table on removal of the mould is measured to assess the quality of concrete.

Depending on the site conditions, the type of concrete, flowable or cohesive in nature, is selected [8].

\section{Air content}

Mortar and concrete mixed with cellulose ether have greatly increased air content, therefore, such antiwashout admixtures contain an air-detraining admixture to reduce the air content of the concrete to between 3 to $5 \%[9]$.

\section{Bleeding}

Concrete containing anti-washout admixture retains more of the mixing water. Because the normal amount of admixture used is more than double the amount required to prevent bleeding, thus very little bleeding occurs in anti-washout UWC. This lack of bleeding is responsible for the small reduction in quality of the concrete [5].

\section{Setting time}

Anti-washout admixtures contain an accelerating admixture, hence the setting time is greatly extended. Anti washout admixtures containing acrylics have no effect on the setting time.The most common accelerating admixture amounts are adjusted to result in a final setting time of from 5 to 12 hours.

\section{Underwater Dispersion Resistance}

The dispersion resistance of concrete during an underwater placement operation is evaluated by tests as: (a) cementitious materials outflow rate, (b) change of water permeation rate, (c) the turbidity of water, (d) the change of $\mathrm{pH}$ value, (e) the change of composition. The rate of dispersion is decreased as the quantity of anti washout admixtures in the underwater concrete is increased.

\section{References}

[1]. John Peter Oleson, et al., The ROMACONS Project: a Contribution to the Historical and Engineering Analysis of Hydraulic Concrete in Roman Maritime Structures, The international journal of Nautical archeology, (Oct 2004) ISSN 1057-2414

[2]. US (United States Army Corps of Engineers) CRD -C61-89A, Test method for determining the resistance of freshly-mixed concrete to washing out in water. US Army Experiment Station,Vicksburg, MS, 1989, pp. 1-3.

[3]. Report on 'Research team recreates ancient underwater concrete technology' dated April 7 2005, University of Colorado at Boulder

[4]. CRD C61, ' Test Methods for Determining the Resistance of Freshly-Mixed Concrete to washing out in Water',US Army Experimentation Station, Handbook on Concrete, Vicksburg, Mississippi, Dec. 1989

[5]. M. Sonebi, P. J. M Bartos, K. H Khayat,' Assessment of washout resistance of underwater concrete: a comparison between CRD C61 and new MC-1 tests' Materials and Structures, Vol.32, May 1999, pp 273-281

[6]. Ceza, M. and Bartos, P.J.M.,'Development of apparatus for testing the washout resistance of underwater concrete mixtures; ACI Concrete in 'Marine Environment', Proceedings Third CANMET/ACI International Conference, SP-163, (V.M. Malhotra, Canada,1996) 111-126

[7]. Khayat, K. H., (1995), Effects of Anti-washout Admixtures on Fresh Concrete Properties, ACJ Materials Journal, 92(2), pp 164171.

[8]. Heniegal, A .M., Maaty, S .A. and Agwa, I. S, (2015), Simulation of the behavior of pressurized underwater concrete, Alexandria Engineering Journal, Faculty of Engineering, Alexandria University54, pp 183-195.

[9]. Assaad, J.J., Daou, Y., and Salman, H., (2011),Correlating washout to strength loss of underwater concrete. Institution of Civil Engineers.J. 106(6), pp 529-536. 